\title{
ANALYTIC AND ENTIRE VECTORS FOR REPRESENTATIONS OF LIE GROUPS
}

\author{
BY \\ ROE GOODMAN( $\left.{ }^{1}\right)$
}

Introduction. The subject of infinite-dimensional representations of Lie groups may be viewed as a generalization of classical harmonic analysis, i.e. the Fourier series and integral. One may ask, then, for the counterpart of Fourier analysis in the complex domain in this general setting.

Two viewpoints seem possible here, group-theoretically. On the one hand, the Fourier integral in the real domain involves the unitary representations $\xi \rightarrow e^{i x \xi}$, $x$ real, of $\boldsymbol{R}$. These representations depend analytically on $x$, of course, and nonreal values of $x$ give nonunitary representations. This process of analytic continuation in the parameters of the representation has been intensively studied for semisimple Lie groups and has led to interesting results concerning harmonic analysis on these groups (cf. [28]).

The second viewpoint, which we shall adopt in the present paper, is to consider the Fourier transform in the complex domain as providing the analytic continuation of a representation of the group $\boldsymbol{R}$ to its complexification $\boldsymbol{C}$.

To be specific, let $U$ be the regular representation of $\boldsymbol{R}$ on $L_{2}(R): U(t) f(x)$ $=f(x+t)$. Suppose $f \in L_{2}(R)$ and

$$
\int_{-\infty}^{\infty} e^{2 r|\xi|}|\hat{f}(\xi)|^{2} d \xi<\infty
$$

for some $r>0(\hat{f}=$ Fourier transform of $f)$. Then $f$ is the restriction to the real axis of the function $f(z)=\int e^{i z \xi} \hat{f}(\xi) d \xi$, which is analytic in the strip $|\operatorname{Im} z|<r$. By setting $U(z) f(x)=f(x+z), \quad|\operatorname{Im} z|<r$, we obtain a local representation of the additive group $\boldsymbol{C}$ on a subspace of $L_{2}(\boldsymbol{R})$.

Now the space of functions satisfying $(0.1)$ ( $r$ depending on $f$ ) is exactly the space of analytic vectors for the representation $U$, i.e. those functions $f$ such that $t \rightarrow U(t) f$ is an analytic function from $\boldsymbol{R}$ to $L_{2}(\boldsymbol{R})$. Furthermore, Paley and Wiener [22] gave still another description of this space as the set of functions $f$, holomorphic in some strip $|\operatorname{Im} z|<r(r$ depending on $f)$ and satisfying

$$
\sup _{|y|<r} \int_{-\infty}^{\infty}|f(x+i y)|^{2} d x<\infty .
$$

Received by the editors December 20, 1968.

(1) This research was supported in part by the Air Force Office of Scientific Research and in part by National Science Foundation grant GP-7952X. 
Suppose now that $\pi$ is a strongly continuous unitary representation of a Lie group $G$ on a Hilbert space $\mathscr{H}=\mathscr{H}(\pi)$. Of course the notion of analytic vector makes sense in this general context. What are the analogues of properties $(0.1)$ and (0.2)? As to (0.1), we observe that this is equivalent to the assertion that $f$ is an analytic vector for the operator $B=\left[1-(d / d x)^{2}\right]^{1 / 2}$ (defined by $(B f)^{\wedge}(\xi)=$ $\left.\left(1+\xi^{2}\right)^{1 / 2} \hat{f}(\xi)\right)$. This operator, however, is definable in general: Let $\mathfrak{g}$ be the Lie algebra of $G$, and let $\partial \pi$ denote the corresponding representation of the universal enveloping algebra of $\mathfrak{g}$ on the space $\mathscr{H}^{\infty}(\pi)$ of $C^{\infty}$ vectors for $\pi$. Choose a basis $X_{1}, \ldots, X_{i}$ for $g$ and set $\Delta=X_{1}^{2}+\cdots+X_{d}^{2}, A=\partial \pi(1-\Delta)^{-}$(the bar denoting operator closure). The operator $A$ is always selfadjoint and positive, by a result of Nelson and Stinespring [21], and for the operator $B=A^{1 / 2}$ we proved [9]

THEOREM A. The space $\mathscr{H}^{\omega}(\pi)$ of analytic vectors for $\pi$ is precisely the space of analytic vectors for the operator $B$.

Thus the analogue of $(0.1)$ is obtained by replacing the Fourier transform by the unitary operator diagonalizing the selfadjoint operator $B$.

It remains to find an analogue of the Paley-Wiener characterization (0.2), viewed as an "intrinsic" description of the space $\mathscr{H}^{\omega}(\pi)$, and to investigate the analytic continuation of $\pi$ to the complexification of $G$. This is the aim of the present paper.

The paper is divided into two parts: Part I, in which we establish certain general results about spaces of analytic vectors, and Part II, in which we make a detailed study of the analytic vectors for representations of certain Lie groups. Our results may be summarized as follows:

In $\S 1$ we prove a theorem which facilitates the determination of the space $\mathscr{H}^{\infty}(\pi)$ of differentiable vectors. In $\S 2$ we introduce a continuous scale $\mathscr{H}_{t}^{\omega}(\pi), 0<t<\infty$, of spaces of analytic vectors, whose union is the space $\mathscr{H}^{\omega}(\pi)$ and whose intersection we call the space of entire vectors for $\pi$. We prove that on the space of entire vectors the representation may be analytically continued to a global representation of the complexified group. In $\S 3$ we prove that when $G$ is the semidirect product of subgroups $H$ and $K$, then $\mathscr{H}^{\omega}(\pi)=\mathscr{H}^{\omega}\left(\left.\pi\right|_{H}\right) \cap \mathscr{H}^{\omega}\left(\left.\pi\right|_{K}\right)$. For a solvable group $G$ this reduces the determination of the space $\mathscr{H}^{\omega}(\pi)$ to the problem of finding the analytic vectors for a one-parameter group, which we treat in $\S 4$.

Part II begins with $\S 5$, in which we recast the Paley-Wiener theorem mentioned earlier into the form of a description of the spaces $\mathscr{H}_{t}^{\omega}(U), U$ the regular representation of $\boldsymbol{R}$. In $\$ 6$ we study the analytic vectors for the irreducible, infinite-dimensional unitary representations of the Heisenberg group. Using Theorem A and the results of Part I we obtain a characterization of Paley-Wiener type for the analytic vectors for these representations. Our result is essentially equivalent to a theorem of Hille concerning Hermite series in the complex domain [11], although our method of proof is completely different. We also characterize the space of entire vectors for these representations and show that it is dense. 
In $\$ 7$ we study the infinite-dimensional irreducible unitary representations of the " $a x+b$ " group, obtaining a theorem of Paley-Wiener type for the "Lebedev transform." Unlike the representations of the Heisenberg group, these representations do not admit any nonzero entire vectors, and we obtain an explicit upper bound for the region of holomorphy of the matrix elements. In $\S 8$ we use the results of $\S 7$ to show that a nowhere-trivial unitary representation of a noncompact simple Lie group $G$ admits no nonzero entire vectors. As corollaries we obtain the existence of complex singularities for spherical functions, and the theorem that $G$ has no nontrivial representations which are continuous in the uniform operator topology, a result due to Singer [27]. These results show that there is no reasonable global form of the "unitarian trick" available for unitary representations of $G$. They also show the essentially local nature of the analytic domination theorem in [20] and [9].

The results of $\S \S 1-3$ are valid for representations on arbitrary Banach spaces, so we establish them in that generality. Thus in Part $I, \pi$ will denote a strongly continuous representation of a connected Lie group $G$ on a complex Banach space $\mathscr{H}(\pi)$. For other treatments of some of the material of $\S \S 1-2$, cf. [2], [4], [5], [18]. Our point of view derives principally from [20].

\section{Part I. General Results}

1. $C^{\infty}$ vectors. We denote by $\mathscr{H}^{\infty}(\pi)$ the space of infinitely differentiable vectors for $\pi$, i.e. those vectors $v \in \mathscr{H}(\pi)$ for which the $\mathscr{H}$-valued function $g \mapsto \tilde{v}(g) \equiv \pi(g) v$ is $C^{\infty}$ on $G$. If $\mathfrak{g}$ is the Lie algebra of $G$, then on the space $\mathscr{H}^{\infty}(\pi)$ there is a representation $\partial \pi$ of $\mathfrak{g}$ such that $\partial \pi(X) v=\left.(d / d t) \pi(\exp t X) v\right|_{t=0}$. The representation $\partial \pi$ extends uniquely to a representation of the universal enveloping algebra $\mathfrak{U}$ of the complexification $\mathfrak{g}^{c}$ of $\mathfrak{g}$, which we shall also denote by $\partial \pi$ (following the notation of [2]).

We topologize $\mathscr{H}^{\infty}(\pi)$ so that $\partial \pi(T)$ is a continuous map, for every $T \in \mathfrak{U}$. For this purpose it suffices to use the countable family of seminorms $\rho_{n}$,

$$
\rho_{n}(v)=\sup _{1 \leqq j_{k} \leqq d}\left\|\partial \pi\left(X_{j_{1}} \cdots X_{j_{n}}\right) v\right\|
$$

where $X_{1}, \ldots, X_{d}$ is a fixed basis for $\mathfrak{g}$ and $n=0,1,2, \ldots\left(\rho_{0}(v)=\|v\|\right)$. These particular seminorms are especially convenient in estimating the rate of growth of $\partial \pi\left(X^{m}\right) v, X \in \mathfrak{g}^{c}$. Indeed, if $X=\sum_{k=1}^{d} \xi_{k} X_{k}$ and we define $|X|=\sum\left|\xi_{k}\right|$ (relative to the fixed basis $\left.\left\{X_{k}\right\}\right)$, then for $v \in \mathscr{H}^{\infty}(\pi)$

$$
\rho_{n}\left(\partial \pi\left(X^{m}\right) v\right) \leqq|X|^{m} \rho_{n+m}(v) .
$$

For $X \in \mathfrak{g}, t \mapsto \pi(\exp t X)$ is a strongly continuous one-parameter group of operators on $\mathscr{H}$ whose infinitesimal generator will be denoted by $d \pi(X)$. Thus $d \pi(X) \supseteq \partial \pi(X)$ and if $v \in \mathscr{H}^{\infty}(\pi)$ then $v \in \mathscr{D}\left(d \pi(X)^{n}\right)$ for all $n$ and all $X \in g(\mathscr{D}(A)$ being the domain of an operator $A$ ). Conversely, we have the following result: 
THEOREM 1.1. Let $X_{1}, \ldots, X_{d}$ be a basis for g. Suppose $v \in \mathscr{H}(\pi)$ is in the domain of $d \pi\left(X_{k}\right)^{n}$ for $n=1,2, \ldots$ and $1 \leqq k \leqq d$. Then $v \in \mathscr{H}^{\infty}(\pi)$.

REMARK. Theorem 1.1 can also be stated in terms of the generating family of one-parameter subgroups $G_{k}=\left\{\exp t X_{k}\right\}:$ If $v$ is a $C^{\infty}$ vector for $\left.\pi\right|_{G_{k}}, k=1, \ldots, d$, then $v$ is $a C^{\infty}$ vector for $\pi$. [One easily verifies that $\mathscr{H}^{\infty}\left(\left.\pi\right|_{G_{k}}\right)=\bigcap_{n=1}^{\infty} \mathscr{D}\left(d \pi\left(X_{k}\right)^{n}\right)$.]

Proof of Theorem 1.1 (suggested by [3]). Set $\tilde{v}_{k}(t)=\pi\left(\exp t X_{k}\right) v$. Then, as noted in the remark above, $\tilde{v}_{k}$ is a $C^{\infty}$ function from $\boldsymbol{R}^{1}$ to $\mathscr{H}$, and $\left.(d / d t)^{n} \tilde{v}_{k}(t)\right|_{t=0}$ $=d \pi\left(X_{k}\right)^{n} v$. Suppose that $\phi \in C_{0}^{\infty}(G)$ and $w \in \mathscr{H}^{\prime}$. Denoting a right Haar measure on $G$ by $d g$, one has

$$
\begin{aligned}
\int \phi(g)\left\langle\pi(g) d \pi\left(X_{k}\right)^{n} v, w\right\rangle d g & =\left.(d / d t)^{n} \int \phi(g)\left\langle\pi\left(g \exp t X_{k}\right) v, w\right\rangle d g\right|_{t=0} \\
& =\left.(d / d t)^{n} \int \phi\left(g \exp -t X_{k}\right)\langle\pi(g) v, w\rangle d g\right|_{t=0} \\
& =(-1)^{n} \int \tilde{X}_{k}^{n} \phi(g)\langle\pi(g) v, w\rangle d g,
\end{aligned}
$$

where $\tilde{X}$ is the left-invariant differential operator $\tilde{X} \phi(g)=\left.(d / d t) \phi(g \exp t X)\right|_{t=0}$.

Now let $\Delta_{m}$ be the differential operator $\tilde{X}_{1}^{2 m}+\cdots+\tilde{X}_{d}^{2 m}$ on $G$. The above calculation shows that for each $w \in \mathscr{H}^{\prime}$ the function $f(g)=\langle\pi(g) v, w\rangle$ is a weak solution to the equation $\Delta_{m} f=f_{m}$, where $f_{m}(g)=\sum_{k=1}^{d}\left\langle\pi(g) d \pi\left(X_{k}\right)^{2 m} v, w\right\rangle$. But $f_{m}$ is a continuous function and $\Delta_{m}$ is an elliptic operator of order $2 m$, so by the local regularity theorem for weak solutions of elliptic equations, $f$ has locally $L_{2}$ derivatives of order $\leqq 2 m$ [1, p. 190, Theorem 1]. Since this holds for all $m, f$ is $C^{\infty}$ by Sobolev's lemma. It follows from [18, Chapter 6, Lemma 1] that $v$ is a $C^{\infty}$ vector for $\pi$. Q.E.D.

COROLlaRY 1.1. The space $\mathscr{H}^{\infty}(\pi)$ is a Fréchet space (metrizable, complete, locally convex).

Proof. Because the topology of $\mathscr{H}^{\infty}(\pi)$ is determined by the countable family $\left\{\rho_{n}\right\}$ of seminorms, we only need show completeness. But if $\left\{v_{n}\right\}$ is a Cauchy sequence in $\mathscr{H}^{\infty}(\pi)$, then for $X \in \mathfrak{g}$ and all $k,\left\{d \pi(X)^{k} v_{n}\right\}_{n=1}^{\infty}$ is a Cauchy sequence in $\mathscr{H}(\pi)$. Let $v=\lim v_{n}$. Because $d \pi(X)$ is a closed operator, we must have $v \in \mathscr{D}\left(d \pi(X)^{k}\right)$ for all $k$. By Theorem 1.1, $v \in \mathscr{H}^{\infty}(\pi)$.

2. Analytic vectors. Recall that the vector $v \in \mathscr{H}$ is an analytic vector for the representation $\pi$ if the function $\tilde{v}(g)=\pi(g) v$ is a real analytic function on $G$. Denoting the space of analytic vectors for $\pi$ by $\mathscr{H}^{\omega}(\pi)$, we have the inclusion $\mathscr{H}^{\omega}(\pi) \subseteq \mathscr{H}^{\infty}(\pi)$. Furthermore, Nelson gave the following infinitesimal characterization of $\mathscr{H}^{\omega}(\pi)$ : Let $X_{1}, \ldots, X_{d}$ be a basis for $g$ which will remain fixed in this section, and define the seminorms $\rho_{n}$ by formula (1.1). Then a $C^{\infty}$-vector $v$ is an analytic vector if and only if

$$
E_{s}(v) \equiv \sum_{n=0}^{\infty} \frac{s^{n}}{n !} \cdot \rho_{n}(v)<\infty
$$


for some $s>0$ [20, Lemma 7.1]. Thus if we define

$$
\mathscr{H}_{t}^{\omega}=\left\{v \in \mathscr{H}^{\infty}(\pi) \mid E_{s}(v)<\infty \text { for } 0<s<t\right\},
$$

then $\mathscr{H}^{\omega}(\pi)=\bigcup_{t>0} \mathscr{H}_{t}^{\omega}$.

The space $\mathscr{H}_{t}^{\omega}$ depends on the choice of basis for $g$ used in defining $\rho_{n}$, of course. If $Y_{1}, \ldots, Y_{d}$ is another basis for $g$ and $\tilde{\mathscr{H}}_{t}^{\omega}$ is defined analogously to $\mathscr{H}_{t}^{\omega}$ but using the basis $\left\{Y_{j}\right\}$, then one easily verifies that $\mathscr{H}_{t / c}^{\omega} \subseteq \tilde{\mathscr{H}}_{t}^{\omega} \subseteq \mathscr{H}_{c t}^{\omega}$ for some $c>0$ and all $t$. Give $\mathscr{H}_{t}^{\omega}$ the topology defined by the seminorms $\left\{E_{s}\right\}_{0<s<t}$.

If $U$ is a unitary representation of $G$, let $A=1-\partial U(\Delta)^{-}$, where $\Delta=X_{1}^{2}+\cdots+X_{d}^{2}$, and set $B=A^{1 / 2}$. Make $\mathscr{D}\left(e^{t B}\right)$ a Hilbert space with norm $\left\|e^{t B} v\right\|$. The proof of Theorem 2 of [9] (cf. especially the estimates of Lemma 2 of [9] and Lemma 6.3 of [20]) establishes the following more precise form of the basic analytic domination theorem quoted in the Introduction:

THEOREM 2.1. There exists a constant $c>0$, independent of $U$, such that for $0<t \leqq 1$

$$
\mathscr{H}_{t / c}^{\omega}(U) \subseteq \mathscr{D}\left(e^{t B}\right) \subseteq \mathscr{H}_{c t}^{\omega}(U)
$$

the inclusions being continuous (uniformly in $U$ ).

Since $\mathscr{D}\left(e^{t B}\right)$ is dense in $\mathscr{H}(U)$ for every $t>0$, Theorem 2.1 shows that $\mathscr{H}_{t_{0}}^{\omega}(U)$ is dense in $\mathscr{H}(U)$ for some $t_{0}>0$, a result due to Nelson. In general for a representation $\pi$ on a Banach space one has $\mathscr{H}^{\omega}(\pi)$ dense in $\mathscr{H}(\pi)$, by [20]. Now from inequality (1.2) it follows that $\mathscr{H}_{t}^{\omega}(\pi)$ is invariant under $\mathfrak{g}$. Hence the closure of $\mathscr{H}_{t}^{\omega}(\pi)$ in $\mathscr{H}(\pi)$ is invariant under $G$ [5, Proposition 1], so that if $\pi$ is topologically irreducible, $\mathscr{H}_{t_{0}}^{\omega}(\pi)$ must be dense in $\mathscr{H}(\pi)$ for some $t_{0}$ in this case also.

The next two propositions state some properties of the spaces $\mathscr{H}_{t}^{\omega}$ which are easily verified consequences of inequality (1.2) and Corollary 1.1.

Proposition 2.1. $\mathscr{H}_{t}^{\omega}$, topologized by the family of norms $\left\{E_{s}\right\}_{0<s<t}$, is a Fréchet space and the inclusion $\mathscr{H}_{t}^{\omega} \subset \mathscr{H}^{\infty}(\pi)$ is continuous. Furthermore, $\partial \pi(X): \mathscr{H}_{t}^{\omega} \rightarrow \mathscr{H}_{t}^{\omega}$ continuously for any $X \in \mathfrak{g}$.

Proposition 2.2. Let $X \in \mathfrak{g}^{c}$ and $v \in \mathscr{H}_{t}^{\omega}$ with $t>|X|$. Then the series

$$
\exp \partial \pi(X) v \equiv \sum_{n=0}^{\infty} \frac{1}{n !} \partial \pi\left(X^{n}\right) v
$$

converges absolutely in $\mathscr{H}^{\infty}(\pi)$ and $\exp \partial \pi(X): \mathscr{H}_{t}^{\omega} \rightarrow \mathscr{H}_{t-|X|}^{\omega}$ continuously. If $X \in \mathfrak{g}$ then $\exp \partial \pi(X) v=\pi(\exp X) v$.

Corollary 2.1. Denote the dual space of $\mathscr{H}^{\infty}(\pi)$ by $\mathscr{H}^{-\infty}(\pi)$. Let $v \in \mathscr{H}_{t}^{\omega}$ and $w \in \mathscr{H}^{-\infty}(\pi)$. Then the function $\phi(X)=\langle\pi(\exp X) v, w\rangle$ on $\mathrm{g}$ extends holomorphically to the region $|X|<t$ in $\mathrm{g}^{c}$.

REMARK. When $\mathscr{H}^{\infty}(\pi)$ is a space of functions on a set $\Gamma$ for which evaluations at points of $\Gamma$ define $\mathscr{H}^{\infty}$-continuous linear functionals, then Corollary 2.1 has the 
consequence that $\pi(\exp X) v(\gamma)$ is holomorphic in $|X|<t$ for each fixed $\gamma \in \Gamma$, whenever $v \in \mathscr{H}_{t}^{\omega}$. (The converse is false, however; cf. §7.)

Let $G^{c}$ be the connected and simply-connected Lie group whose Lie algebra is $\mathrm{g}^{c}$. Fix $t_{0} \leqq 1 / 4$ such that $X \rightarrow \exp X$ is bijective from $\mathrm{g}^{c}$ to $G^{c}$. (Here $\exp =\exp _{G^{c}}$.) Then for $|X|<t_{0}$ and $g=\exp X$, we define $\pi_{\omega}(g)=\exp \partial \pi(X)$, with domain $\bigcup_{t>|X|} \mathscr{H}_{t}^{\omega}$. Let $\mid$ ad $X \mid$ be the norm of ad $X$ as a linear transformation on the normed space $\left(\mathfrak{g}^{c},|\cdot|\right)$ (where $|\cdot|$ is the norm defined by the fixed basis $\left\{X_{k}\right\}$ ). Then we have the following result (cf. [20, Lemma 9.1]):

Proposition 2.3. $\pi_{\omega}$ is a local representation of $G^{c}$ :

If $\max (|X|,|\operatorname{ad} X|,|Y|,|\operatorname{ad} Y|)<t \leqq t_{0}$ and $s=\left(e^{2 t}-1\right) /\left(2-e^{2 t}\right) \equiv \theta(t)$, then for $v \in \mathscr{H}_{s}^{\omega}$

$$
\pi_{\omega}(\exp X) \pi_{\omega}(\exp Y) v=\pi_{\omega}(\exp X \exp Y) v .
$$

Remark. Note that $\theta(t)>2 t$, with $\theta(t) \sim 2 t$ as $t \rightarrow 0$.

Proof. We shall employ the Campbell-Hausdorff formula [12], which is the formal identity $e^{x} e^{y}=e^{L(x, y)}$, with

$$
L(x, y)=\sum_{n=1}^{\infty} \frac{(-1)^{n}}{n} \sum_{(p, q) \in S_{n}} c(p, q) Z_{p, q}(x, y) .
$$

Here $S_{n}=\left\{(p, q) \mid p, q \in N^{n}, p_{i}+q_{i}>0\right\}, c(p, q)=[p ! q !|p+q|]^{-1}$, and

$$
\begin{aligned}
& Z_{p, q}(x, y)=(\operatorname{ad} x)^{p_{1}}(\operatorname{ad} y)^{q_{1}} \cdots(\operatorname{ad} x)^{p_{n}}(\operatorname{ad} y)^{q_{n}-1}(y) \text { if } q_{n}>0,
\end{aligned}
$$

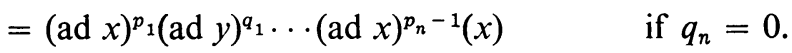

(We employ the usual notation: if $p \in N^{n}$, then $p !=p_{1} ! \cdots p_{n}$ ! and $|p|=p_{1}+\cdots$ $+p_{n}$.)

Let us investigate the convergence properties of the series $L(\partial \pi(X), \partial \pi(Y))$. Because $\partial \pi$ is a representation, $Z_{p q}(\partial \pi(X), \partial \pi(Y))=\partial \pi\left(Z_{p q}(X, Y)\right)$. By our assumptions on $X$ and $Y$, we have $\left|Z_{p q}(X, Y)\right|<t^{|p|+|q|}$. Thus for $v \in \mathscr{H}^{\infty}(\pi)$ and $m \geqq 0$, $\rho_{m}\left(Z_{p q}(\partial \pi(X), \partial \pi(Y)) v\right) \leqq t^{|p|+|q|} \rho_{m+1}(v)$, by inequality (1.2). But

$$
\sum_{n=1}^{\infty} \frac{1}{n} \sum_{p, q \in S_{n}} c(p, q) t^{|p|+|q|}<\sum_{n=1}^{\infty}\left(e^{2 t}-1\right)^{n}=\theta(t)
$$

the series for $\theta(t)$ converging by virtue of the restriction $t \leqq 1 / 4$. Hence $|L(X, Y)|$ $<\theta(t)$ and the series $L(\partial \pi(X), \partial \pi(Y)) v$ converges absolutely in $\mathscr{H}^{\infty}(\pi)$ to $\partial \pi(L(X, Y)) v$.

Suppose now that $v \in \mathscr{H}_{\theta(t)}^{\omega}$. Since $\theta(t)>2 t, \pi_{\omega}(\exp Y) v \in \mathscr{H}_{t}^{\omega}$ and the series defining $\pi_{\omega}(\exp X) \pi_{\omega}(\exp Y) v$ is absolutely convergent by Proposition 2.2. The series defining $\pi_{\omega}(\exp L(X, Y)) v$ converges absolutely by the estimates of the preceding paragraph. Hence these series are all unconditionally convergent, so the Campbell-Hausdorff formula applies, yielding

$$
\pi_{\omega}(\exp X) \pi_{\omega}(\exp Y) v=\pi_{\omega}(\exp L(X, Y)) v=\pi_{\omega}(\exp X \exp Y) v
$$

[12, Chapter X, Theorem 3.1]. Q.E.D. 
Let us set $\mathscr{H}_{\infty}^{\omega}(\pi)=\bigcap_{t>0} \mathscr{H}_{t}^{\omega}$. By an earlier remark this space is independent of the choice of basis for $g$ used to define $\mathscr{H}_{t}^{\omega}$. By Proposition 2.1 it is a Fréchet space when topologized by the family of norms $\left\{E_{r}\right\}_{r>0}$. Furthermore, if $v \in \mathscr{H}_{\infty}^{\omega}(\pi)$ and $X \in \mathfrak{g}^{c}$, then $\exp \partial \pi(X) v \in \mathscr{H}_{\infty}^{\omega}(\pi)$ and the function $X \rightarrow \exp \partial \pi(X) v$ is holomorphic, by Proposition 2.2. We shall call $\mathscr{H}_{\infty}^{\omega}(\pi)$ the space of entire vectors for $\pi$. As an immediate consequence of Propositions 2.3 and the simple-connectivity of $G^{c}$, we thus obtain

Corollary 2.2. $\pi_{\omega}$ defines a holomorphic representation of $G^{c}$ on the space $\mathscr{H}_{\infty}^{\omega}(\pi)$

REMARK. Suppose $\pi$ is topologically irreducible. Then, as a space of analytic vectors invariant under $\mathfrak{g}, \mathscr{H}_{\infty}^{\omega}(\pi)$ is either 0 or dense in $\mathscr{H}(\pi)$. We shall give examples of both possibilities in $\$ \S 6$ and 7 .

3. Analytic vectors for semidirect products. In $\S 1$ we saw that the determination of the space $\mathscr{H}^{\infty}(\pi)$ could be reduced to the case of one-parameter subgroups. We would like to obtain some similar reduction of the problem of characterizing the space $\mathscr{H}^{\omega}(\pi)$. The situation here is more delicate, since separate real-analyticity of a function of several variables does not imply joint analyticity. Thus the analogue of Theorem 1.1, with " $C^{\infty}$ " replaced by " analytic," may not hold. We do have the following theorem, however.

THEOREM 3.1. Let $G$ be the semidirect product of subgroups $H$ and $K$, and let $\pi_{H}$ and $\pi_{K}$ denote, respectively, the restrictions of $\pi$ to $H$ and $K$. Then

$$
\mathscr{H}^{\omega}(\pi)=\mathscr{H}^{\omega}\left(\pi_{H}\right) \cap \mathscr{H}^{\omega}\left(\pi_{K}\right)
$$

Proof. Let us suppose $K$ normal in $G$, and denote by $\mathfrak{h}$ and $\mathfrak{f}$ the Lie algebras of $H$ and $K$ respectively. Then $\mathfrak{g}=\mathfrak{h}+\mathfrak{t}$ (direct sum), and $\operatorname{Ad} G(\mathfrak{l}) \subseteq \mathfrak{f}$ (Ad being the adjoint representation of $G$ on $\mathfrak{g})$. Let $X_{1}, \ldots, X_{d}$ be a basis for $\mathfrak{g}$ such that $X_{j} \in \mathfrak{f}$ for $1 \leqq j \leqq l$, and $X_{j} \in \mathfrak{h}$ for $l+1 \leqq j \leqq d$. The map $\tau \rightarrow e(\tau) \equiv \exp t_{1} X_{1} \cdots \exp t_{d} X_{d}$, where $\tau=\left(t_{1}, \ldots, t_{d}\right) \in \boldsymbol{R}^{d}$, is an analytic isomorphism from a neighborhood of 0 in $\boldsymbol{R}^{d}$ to a neighborhood of $e$ in $G$ [12, Chapter VII, Proposition 4.1]. Hence $v \in \mathscr{H}^{\omega}(\pi)$ if and only if the function $F(\tau)=\pi(e(\tau)) v$ is analytic for $\tau$ near zero in $\boldsymbol{R}^{d}$.

Suppose that $v \in \mathscr{H}^{\omega}\left(\pi_{H}\right) \cap \mathscr{H}^{\omega}\left(\pi_{K}\right)$. Then $v$ is a $C^{\infty}$ vector for each oneparameter group $\left\{\exp t X_{k}\right\}$, so by Theorem $1.1 v \in \mathscr{H}^{\infty}(\pi)$. Thus the function $F(\tau)$ is $C^{\infty}$. To prove $F$ analytic we must show that there exists an $\varepsilon>0$ and $M<\infty$ such that the Cauchy inequalities

$$
\left\|D^{\alpha} F(\tau)\right\| \leqq M^{|\alpha|}|\alpha| !
$$

hold for $|\tau| \leqq \varepsilon$ and all multi-indices $\alpha=\left(\alpha_{1}, \ldots, \alpha_{d}\right)$, where $D^{\alpha}=D_{1}^{\alpha_{1}} \ldots D_{d}^{\alpha_{d}}$ $\left(D_{j}=\partial / \partial t_{j}\right)$. One calculates easily that

$$
D_{j}^{k} F(\tau)=\pi\left(\exp t_{1} X_{1} \cdots \exp t_{j} X_{j}\right) \partial \pi\left(X_{j}\right)^{k} \pi\left(\exp t_{j+1} X_{j+1} \cdots \exp t_{d} X_{d}\right) v .
$$


But for $v \in \mathscr{H}^{\infty}(\pi), g \in G$, and $X \in \mathfrak{g}$, one has the relation

$$
\partial \pi(X)^{k} \pi(g) v=\pi(g) \partial \pi\left(\operatorname{Ad} g^{-1} \cdot X\right)^{k} v,
$$

obtained by differentiating the equation $g^{-1} \exp t X g=\exp \left(\operatorname{Ad} g^{-1} \cdot t X\right) \quad[10$, Chapter II, §5]. Thus $D_{j}^{k} F(\tau)=\pi(e(\tau)) \partial \pi\left(Z_{j}(\tau)\right)^{k} v$, where

$$
Z_{j}(\tau)=\operatorname{Ad}\left(\exp t_{j+1} X_{j+1} \cdots \exp t_{d} X_{d}\right)^{-1} \cdot X_{j}
$$

Since $\pi$ is uniformly bounded on compact sets, one has

$$
\sup _{|\tau| \leqq \varepsilon}\left\|D_{j}^{k} F(\tau)\right\| \leqq C_{\varepsilon} \sup _{|\tau| \leqq \varepsilon}\left\|\partial \pi\left(Z_{j}(\tau)\right)^{k} v\right\|
$$

holding for any $\varepsilon>0$ and some constant $C_{\varepsilon}$. But by the choice of the basis $\left\{X_{j}\right\}$ we have $Z_{j}(\tau) \in \mathfrak{h}$ for $j \geqq l+1$ while $Z_{j}(\tau) \in \mathfrak{f}$ for $j \leqq l$. Since $v \in \mathscr{H}^{\omega}\left(\pi_{H}\right) \cap \mathscr{H}^{\omega}\left(\pi_{K}\right)$ and the map $\tau \rightarrow Z_{j}(\tau)$ is continuous, there exists an $\varepsilon>0$ and $M<\infty$ so that $\left\|\partial \pi\left(Z_{j}(\tau)\right)^{k} v\right\| \leqq M^{k} k$ ! for $|\tau| \leqq \varepsilon$. Thus we get

$$
\sup _{|\tau| \leqq \varepsilon}\left\|D_{j}^{k} F(\tau)\right\| \leqq M^{k} k !
$$

holding for some $\varepsilon>0$ and $M<\infty$.

We now want to employ a theorem of F. Browder [3]. Since the proof of this theorem in [3] has a small technical error (the function $\zeta_{r}$ on page 653 is obviously not of class $C^{r+1}$ as claimed), we shall sketch a proof incorporating the necessary corrections.

Set $A_{m}=1-\sum_{j=1}^{d} D_{j}^{2 m}$. By the argument of [3], one has an estimate

$$
\left\|D^{\alpha} \phi\right\|_{\infty} \leqq C\left\|A_{m} \phi\right\|_{\infty}
$$

holding for $|\alpha|<2 m-d$ and $\phi \in C_{0}^{2 m}\left(\boldsymbol{R}^{d}\right)$ ( $\|\cdot\|_{\infty}$ being sup norm). The constant $C$ depends only on $d$ and the support of $\phi$.

One next constructs a function $\psi_{m}$ of class $C^{m}$ with compact support, which, roughly speaking, satisfies Cauchy's inequalities up to order $m$. For this purpose, define a function $\phi_{m}$ on $\boldsymbol{R}^{1}$ by

$$
\begin{aligned}
\phi_{m}(t) & =0, & & t \leqq 0, \\
& =\int_{0}^{t} s^{m}(1-s)^{m} d s, & & 0 \leqq t \leqq 1, \\
& =\phi_{m}(1), & & t \geqq 1 .
\end{aligned}
$$

The vanishing of the integrand to order $m$ at 0 and 1 implies that $\phi_{m} \in C^{m}\left(\boldsymbol{R}^{1}\right)$. Straightforward estimates with binomial coefficients show that for $k \leqq m$,

$$
\left\|(d / d t)^{k} \phi_{m}\right\|_{\infty} \leqq 4^{m} k ! .
$$

Set $\theta_{m}(t)=\phi_{m}(1)^{-1} \phi_{m}(2-2|t|)$, and

$$
\psi_{m}(\tau)=\prod_{j=1}^{d} \theta_{m}\left(t_{j} / \varepsilon\right), \quad \tau \in \boldsymbol{R}^{d},
$$


$\varepsilon$ as in (3.2). Then $\psi_{m} \in C^{m}\left(\boldsymbol{R}^{d}\right), \psi_{m}(\tau)=0$ for $|\tau| \geqq \varepsilon, \psi_{m}(\tau)=1$ for $|\tau| \leqq \varepsilon / 2$, and $0 \leqq \psi_{m}(\tau) \leqq 1$. Since $\phi_{m}(1) \geqq C^{m}$, it follows that for $k \leqq m$,

$$
\left\|D_{j}^{k} \psi_{m}\right\|_{\infty} \leqq C_{\varepsilon}^{m} k !
$$

$C_{\varepsilon}$ a constant depending only on $\varepsilon$.

We now combine estimates (3.2), (3.3), and (3.4) as follows: Let $u \in \mathscr{H}^{\prime},\|u\|=1$, and set $f(\tau)=\langle F(\tau), u\rangle$. We obtain from (3.3) the estimate

$$
\sup _{|\tau| \leqq \varepsilon / 2}\left|D^{\alpha} f(\tau)\right| \leqq C_{\varepsilon} \sup _{|\tau| \leqq \varepsilon}\left|A_{m}\left(\psi_{2 m} f\right)(\tau)\right|
$$

for any multi-index $\alpha$ with $|\alpha|<2 m-d\left(C_{\varepsilon}\right.$ is independent of $m$ since $\psi_{2 m} f$ is supported on the cube $|\tau| \leqq \varepsilon$ for all $m$ ). By Leibnitz' formula and estimates (3.2) and (3.4) we obtain

$$
\sup _{|\tau| \leqq \varepsilon}\left|D_{j}^{2 m}\left(\psi_{2 m} f\right)(\tau)\right| \leqq M_{1}^{2 m}(2 m) !
$$

for some constant $M_{1}$. Together with (3.5), this gives

$$
\sup _{|\tau| \leqq \varepsilon / 2}\left|D^{\alpha} f(\tau)\right| \leqq M_{2}^{2 m}(2 m) !
$$

holding for some constant $M_{2}$ and any multi-index $\alpha$ with $|\alpha|<2 m-d$. Choose $m$ so that $2 m \leqq d+|\alpha|+2$. Then the Cauchy inequalities (3.1) follow from (3.6) and the arbitrariness of $u$. Q.E.D.

Theorem 3.1 yields the analytic counterpart to Theorem 1.1 in the solvable case:

Corollary 3.1. Suppose $G$ is a solvable Lie group. Choose a basis $\left\{X_{j}\right\}_{j=1}^{d}$ for $\mathrm{g}$ such that $\mathfrak{L}_{i} \equiv \operatorname{span}\left\{X_{j}\right\}_{j \leqq i}$ is a subalgebra of $\mathrm{g}$ with $\mathfrak{L}_{i}$ an ideal in $\mathfrak{L}_{i+1}$. Let $G_{j}=$ $\left\{\exp t X_{j}\right\}_{-\infty<t<\infty}$. Then $\mathscr{H}^{\omega}(\pi)=\bigcap_{j=1}^{d} \mathscr{H}^{\omega}\left(\left.\pi\right|_{G_{j}}\right)$.

Proof. We may assume $G$ connected and simply connected, since the notion of analytic vector is a local one. Let $H_{i}$ be the subgroup of $G$ corresponding to the subalgebra $\mathfrak{\Omega}_{i}$. Then by [12, Chapter XII, Theorem 2.2] $H_{i+1}$ is simply connected and is the semidirect product of $H_{i}$ with $G_{i+1}$. The corollary thus follows from Theorem 3.1 by induction on $\operatorname{dim} Y$. Q.E.D.

4. Analytic vectors for a one-parameter unitary group. In order to utilize the results of $\$ 3$ we shall need convenient criteria for determining the analytic vectors for a one-parameter unitary group of operators $t \mapsto U(t)$. By Stone's theorem $U(t)=e^{i t A}$ for a unique selfadjoint operator $A$. By the spectral theorem [14], we may realize $A$ as multiplication by a real-valued measurable function $\phi$ acting on $L_{2}(\Omega, d \omega)$, for some measure space $(\Omega, d \omega)$. We then have the following criteria for analyticity:

Proposition 4.1. Let $f \in L_{2}(\Omega, d \omega)$. The following are equivalent:

(i) $\phi^{n} f \in L_{2}$ for all $n$ and $\sum_{n=0}^{\infty}\left(t^{n} / n !\right)\left\|\phi^{n} f\right\|<\infty$ for $0 \leqq t<r$. 
(ii) $e^{t|\phi|} f \in L_{2}$ for $0 \leqq t<r$.

(iii) The function $F(x)=\left(e^{i x \phi} f, f\right), x$ real, extends holomorphically to the disc $|z|<2 r$.

Proof. (i) $\Rightarrow$ (ii) The series $\sum\left(z^{n} / n\right.$ !) $\phi^{n} f$ converges in $L_{2}$ for $|z|<r$; it also converges pointwise to $e^{z \phi} f$, which is thus in $L_{2}$.

(ii) $\Rightarrow$ (iii) $F(z)=\int e^{i z \phi}|f|^{2} d \omega$ gives the analytic continuation, the integral being absolutely convergent for $|z|<2 r$.

(iii) $\Rightarrow$ (i) Let $u(t)=\operatorname{Re} F(t)=\int \cos (t \phi)|f|^{2} d \omega$. Since $u$ is $C^{\infty}$ near 0 , and $u(t)=u(-t)$, we have $u^{\prime}(0)=0$, so that $\lim _{t \rightarrow 0} t^{-2}[u(0)-u(t)]$ exists. By Fatou's lemma this implies $\int \phi^{2}|f|^{2} d \omega<\infty$, so we may apply the dominated convergence theorem to conclude that

$$
u^{\prime \prime}(t)=-\int \cos (\phi t) \phi^{2}|f|^{2} d \omega .
$$

It follows by induction that $\phi^{n} f \in L_{2}$ for all $n$. With this information, the dominated convergence theorem yields the equality $F^{(2 n)}(0)=(-1)^{n}\left\|\phi^{n} f\right\|^{2}$. But letting $M(s)=\sup _{|z|=s}|F(z)|$, one then has by Cauchy's inequalities $\left\|\phi^{n} f\right\|^{2} \leqq M(s)(2 n) ! s^{-2 n}$ $\leqq M(s)(n !)^{2}(s / 2)^{-2 n}$ for any $s<2 r$, which implies (i). Q.E.D.

\section{Part II. Applications}

5. Analytic vectors for translations. Let $\mathscr{H}=L_{2}\left(\boldsymbol{R}^{1}\right)$ (Lebesgue measure), and let $U(t)$ be the one-parameter unitary group of translations: $U(t) f(x)=f(x+t)$. If $\hat{f}$ denotes the Fourier transform of $f$, then $(U(t) f)^{\wedge}(\xi)=e^{-i t \xi} \hat{f}(\xi)$. Thus with respect to the canonical basis $\{X\}$ for the Lie algebra of $\boldsymbol{R}\left(X f=\left.(d / d t) f(t)\right|_{t=0}\right)$,

$$
\mathscr{H}_{t}^{\omega}(U)=\left\{f \mid e^{s|\xi|} \hat{f}(\xi) \in L_{2} \text { for } s<t\right\},
$$

by Proposition 4.1. This space of functions, however, was characterized intrinsically by Paley and Wiener [22, Theorems I and IV]:

Proposition 5.1 (Paley-Wiener). A function $f$ in $L_{2}\left(\boldsymbol{R}^{1}\right)$ is in the space $\mathscr{H}_{t}^{\omega}(U)$ if and only if it is the restriction to the real axis of a function $f(z)$ which is holomorphic in the strip $|\operatorname{Im} z|<t$ and which satisfies

$$
N_{s}(f) \equiv \sup _{|y| \leqq s}\left\{\int_{-\infty}^{\infty}|f(x+i y)|^{2} d x\right\}^{1 / 2}<\infty
$$

for all $s<t$.

(For a neat proof, cf. [13, Chapter VI, §7].)

COROLlaRY 5.1. The norms $\left\{N_{s}\right\}_{s<t}$ in equation (5.1) define the topology of the space $\mathscr{H}_{t}^{\omega}(U)$. If $f \in \mathscr{H}_{t}^{\omega},|z|<t$, then $U_{\omega}(z) f(x)=f(x+z)$.

Proof of Corollary 5.1. We use the fact that for $f \in \mathscr{H}_{t}^{\omega}(U)$ and $|\operatorname{Im} z|<t$, the Fourier transform of $f(x+z)$ is $e^{z z} \hat{f}(\xi)$ [13, loc. cit.]. In particular one has $N_{s}(f)$ 
$\leqq E_{s}(f) \equiv \sum_{n=0}^{\infty}\left(s^{n} / n !\right)\left\|f^{(n)}\right\|$. But also this gives $U_{\omega}(z) f(x)=f(x+z)$, so $N_{s}(f)$ $=\sup _{|z| \leqq s}\left\|U_{\omega}(z) f\right\|$. Hence by using the Cauchy inequalities applied to the holomorphic vector-valued function $z \rightarrow U_{\omega}(z) f$ we can dominate $E_{s}(f)$ by $N_{r}(f)$, $s<r<t$. Q.E.D.

REMARKS. The space $\mathscr{H}_{\infty}^{\omega}(U)$ of entire vectors for translations is the space of entire functions $f$ for which $N_{s}(f)<\infty$ for all $s>0$; the representation $U_{\omega}$ is given by complex translations. Any analytic vector $f$ for the operator $d^{2} / d x^{2}$ is an entire vector for $U$, since $e^{\varepsilon|\xi|^{2}} \hat{f}(\xi)$ is then in $L_{2}$ for some $\varepsilon>0$. In particular $\mathscr{H}_{\infty}^{\omega}(U)$ is dense in $L_{2}(R)$.

In $\S \S 6$ and 7 we will need the following uniform bounds for functions in $\mathscr{H}^{\omega}(U)$ :

Lemma 5.1. Let $f \in \mathscr{H}_{t}^{\omega}(U)$ and $r<s<t$. Then

$$
\sup _{\| \operatorname{Im} z \mid \leqq r}|f(z)| \leqq \theta(r, s) E_{s}(f),
$$

$\theta(r, s)$ a constant independent of $f$.

Proof. Immediate from the Fourier inversion formula and the relation

$$
(f(x+z))^{\wedge}=e^{z \xi} \hat{f}(\xi), \quad|\operatorname{Im} z|<t .
$$

6. The Heisenberg group. The Heisenberg group is the simply connected nilpotent Lie group $G$ having Lie algebra $g$ spanned by $X, Y, Z$, with commutation relations $[X, Y]=Z, Z$ central. The infinite-dimensional irreducible unitary representations of $G$ map $Z$ into $i \lambda \cdot 1, \lambda \in \boldsymbol{R}, \lambda \neq 0$, and are determined by $\lambda$. The representation $\pi^{\lambda}$ may be realized on $L_{2}(R)$, with $X \rightarrow d / d x, Y \rightarrow i \lambda x$, or in exponentiated form $\exp t X \rightarrow$ translation by $t$, exp $t Y \rightarrow$ multiplication by $e^{i \lambda t x}$ [24, Chapter 2, §1].

It follows from Theorem 1.1 that the space of $C^{\infty}$-vectors for these representations is the Schwartz space $\mathscr{S}$ of rapidly decreasing $C^{\infty}$ functions. Indeed, Theorem 1.1 provides the superficially weaker condition: $f \in \mathscr{S} \Leftrightarrow$ the derivatives $f^{(n)} \in L_{2}$ for all $n$ and $p \cdot f \in L_{2}$ for all polynomials $p$.

Consider now the space $\mathscr{H}^{\omega}\left(\pi^{\lambda}\right)$. Since $\partial \pi^{\lambda}\left(X^{m} Y^{n} Z^{k}\right)=\lambda^{n+k} \partial \pi^{1}\left(X^{m} Y^{n} Z^{k}\right)$, it follows that $\mathscr{H}^{\omega}\left(\pi^{\lambda}\right)$ is independent of $\lambda$. Let $\Delta=X^{2}+Y^{2}+Z^{2}$. Then $\partial \pi^{1}(\Delta)$ $=(d / d x)^{2}-x^{2}-1$, acting on $\mathscr{S}$. As is well-known, this operator has a simple discrete spectrum $\lambda_{n}=-2(n+1)$, with the corresponding normalized eigenfunctions $\phi_{n}$ being the Hermite functions:

$$
\phi_{n}(x)=\left(\pi^{1 / 2} 2^{n} n !\right)^{-1 / 2} \cdot(-1)^{n} e^{x^{2} / 2}(d / d x)^{n}\left(e^{-x^{2}}\right), \quad[17] .
$$

By Theorem A of the Introduction and Proposition 4.1, the space $\mathscr{H}^{\omega}\left(\pi^{\lambda}\right)$ is thus the space of $L_{2}$ functions $f$ whose "Hermite coefficients" $a_{n}=\int_{-\infty}^{\infty} f(x) \phi_{n}(x) d x$ satisfy $\sum \exp (\sigma \sqrt{ } n)\left|a_{n}\right|^{2}<\infty$ for some $\sigma>0$. This last condition is of course equivalent to the condition $a_{n}=O(\exp -\sigma \sqrt{ } n)$ for some $\sigma>0$. Functions satisfying this condition have been characterized in terms of their analytic continuations by 
E. Hille [11, Theorem 1]. We give a slightly weakened version of his result in the next theorem (Hille's theorem correlates the numbers $\sigma, \tau, \varepsilon$ in (ii) and (iii) below):

THeOREM 6.1. Let $f \in L_{2}(R)$ and let $\left\{a_{n}\right\}$ be the "Hermite coefficients" of $f$. The following are equivalent:

(i) $f$ is an analytic vector for the representation $\pi^{\lambda}$ of the Heisenberg group.

(ii) $a_{n}=O(\exp -\sigma \sqrt{ } n)$ for some $\sigma>0$.

(iii) $f$ is the restriction to the real axis of a function $f(z)$ which is holomorphic in a strip $|\operatorname{Im} z|<\tau$ and satisfies

$$
\sup _{|y|<\tau}|f(x+i y)| \leqq C e^{-\varepsilon|x|}
$$

for some positive $\varepsilon, \tau$ and $C<\infty$.

Proof. The equivalence of (i) and (ii) has already been noted. Suppose $f$ satisfies (iii). Then by Proposition 5.1, $f \in \mathscr{H}_{\tau}^{\omega}(U)$, so $f$ is an analytic vector for $\partial \pi^{\lambda}(X)$. By Proposition $4.1 f$ is an analytic vector for $\partial \pi^{\lambda}(Y)$, and trivially $f$ is an analytic vector for $\partial \pi^{\lambda}(Z)$. Hence $f \in \mathscr{H}^{\omega}\left(\pi^{\lambda}\right)$ by Corollary 3.1.

Conversely, suppose $f \in \mathscr{H}^{\omega}\left(\pi^{\lambda}\right)$. Let $E_{t, \lambda}$ be defined by equation (2.1) relative to the representation $\pi^{\lambda}$ and the basis $X, Y, Z$ of $\mathrm{g}$. Let $E_{t}$ be the similar seminorm associated with the representation $U$ of $R$ in $\$ 5$. Since the restriction of $\pi^{\lambda}$ to the subgroup $\{\exp t X\}$ is the representation $U$, we have $\mathscr{H}_{t}^{\omega}\left(\pi^{\lambda}\right) \subseteq \mathscr{H}_{t}^{\omega}(U)$ and $E_{t}(f)$ $\leqq E_{t, \lambda}(f)$. Now $f \in \mathscr{H}_{t}^{\omega}\left(\pi^{\lambda}\right)$ for some $t>0$. Thus by Proposition $5.1 f$ is holomorphic in some strip $|\operatorname{Im} z|<t$. Set $F_{w}(x)=e^{i \lambda w x} f(x)=\exp \left[w \partial \pi^{\lambda}(Y)\right] f(x)$ for $|w|<t$. If $s>0$ and $s+|w|<t$, then by Proposition $2.2 F_{w} \in \mathscr{H}_{t-|w|}^{\omega}\left(\pi^{\lambda}\right)$ and $E_{s}\left(F_{w}\right) \leqq E_{s, \lambda}\left(F_{w}\right)$ $\leqq c(w) E_{s+|w|, \lambda}(f)<\infty$. By Lemma 5.1 we conclude that for $0<r<s<t-|w|$,

$$
\sup _{|\operatorname{Im} z| \leqq r}\left|F_{w}(z)\right| \leqq c(r, s, w),
$$

which yields (iii). Q.E.D.

We now want to make a closer study of the spaces $\mathscr{H}_{t}^{\omega}\left(\pi^{\lambda}\right)$, defined relative to the basis $X, Y, Z$ of $g$. For this purpose let $\mathscr{A}_{t, \lambda}$ be the space of functions $f(z)$ holomorphic in the strip $|\operatorname{Im} z|<t$, such that

$$
M_{r, \lambda}(f) \equiv \sup _{|y| \leqq r ;-\infty<x<\infty} e^{r|\lambda x|}|f(x+i y)|<\infty
$$

for all $r<t$. Topologize $\mathscr{A}_{t, \lambda}$ by the family of norms $\left\{M_{r, \lambda}\right\}_{0<r<t}$.

THEOREM 6.2. For any $t>0$,

$$
\mathscr{H}_{t}^{\omega}\left(\pi^{\lambda}\right) \subseteq \mathscr{A}_{t, \lambda} \supseteq \mathscr{H}_{2 t}^{\omega}\left(\pi^{\lambda}\right),
$$

the inclusions being topological. In particular, the space of entire vectors for $\pi^{\lambda}$ consists of all entire functions $f$ such that $M_{r, \lambda}(f)<\infty$ for all $r>0$, topologized by the family of norms $\left\{M_{r, \lambda}\right\}_{0<r<\infty}$.

REMARK. The families of norms $\left\{M_{r, \lambda_{1}}\right\}_{0<r<\infty}$ and $\left\{M_{r, \lambda_{2}}\right\}_{0<r<\infty}$ are obviously equivalent for any $\lambda_{1}, \lambda_{2} \neq 0$. Hence $\mathscr{H}_{\infty}^{\omega}\left(\pi^{\lambda}\right)$ is in fact independent of $\lambda$. 
Proof. We introduce an "intermediate" family of seminorms $L_{r, \lambda}$ :

$$
L_{r, \lambda}(f)=\sup _{m, n \geqq 0} r^{m+n}(m ! n !)^{-1}\left\|\partial \pi^{\lambda}\left(X^{m} Y^{n}\right) f\right\|,
$$

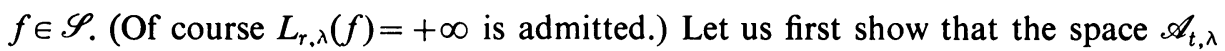
can be defined by the seminorms $\left\{L_{r, \lambda}\right\}_{r<t}$. Indeed, suppose $f \in \mathscr{S}$ and $L_{r, \lambda}(f)<\infty$. Then the power series for $\exp \left[w \partial \pi^{\lambda}(X)\right] \exp \left[v \partial \pi^{\lambda}(Y)\right] f$ is absolutely convergent in $L_{2}$ for $\max (|w|,|v|)<r$. Arguing as in the proof of Theorem 6.1, we conclude that $f$ is holomorphic in the strip $|\operatorname{Im} z|<r$ and $M_{s, \lambda}(f) \leqq c(s, r, \lambda) L_{r, \lambda}(f)$ for $s<r$. Conversely, if $f \in \mathscr{A}_{r, \lambda}$, then by standard arguments using Fubini and Morera's theorems the function $F_{w, v}, F_{w, v}(x)=e^{\lambda w(x+v)} f(x+v)$, is a holomorphic function from $|w|<r,|v|<r$ to $L_{2}$. Furthermore, $\left.(\partial / \partial v)^{m}(\partial / \partial w)^{n} F_{w, v}\right|_{w=v=0}=\partial \pi^{\lambda}\left(X^{m} Y^{n}\right) f$. An application of Cauchy's inequalities thus gives the estimate

$$
L_{t, \lambda}(f) \leqq \sup _{|w|=|v|=t}\left\{\int\left|e^{\lambda w(x+v)} f(x+v)\right|^{2} d x\right\}^{1 / 2} \leqq c(t, s, \lambda) M_{s, \lambda}(f)
$$

for any $0<t<s<r$, with $c(t, s, \lambda)$ independent of $f$.

Given now that $\mathscr{A}_{t, \lambda}=\left\{f \in \mathscr{S} \mid L_{r, \lambda}(f)<\infty\right.$ for $\left.r<t\right\}$, it is trivial to show $\mathscr{A}_{t, \lambda}$ $\supseteq \mathscr{H}_{2 t}^{\omega}\left(\pi^{\lambda}\right)$, with continuous inclusion. It remains to show that the seminorms $\left\{L_{r, \lambda}\right\}_{r<t}$ dominate the seminorms $\left\{E_{r, \lambda}\right\}_{r<t}$. For this purpose we will use the following lemmas:

Lemma 6.1. Let $\alpha_{j}, \beta_{j}$ be nonnegative integers. Then

$$
Y^{\alpha_{1}} X^{\beta_{1}} \ldots Y^{\alpha_{n}} X^{\beta_{n}}=\sum_{k \in N^{n}} c_{\alpha, \beta}(k) Z^{|k|} X^{|\beta-k|} Y^{|\alpha-k|},
$$

where the coefficients $c_{\alpha, \beta}(k)$ satisfy

$$
\left|c_{\alpha, \beta}(k)\right| \leqq \frac{1}{k !} \frac{|\alpha| !}{(|\alpha|-|k|) !} \frac{\beta !}{(\beta-k) !}
$$

$\left(c_{\alpha, \beta}(k)=0\right.$ if $\left.k>\min (\alpha, \beta)\right)$.

Proof of Lemma 6.1. We start from the identity

$$
Y^{m} X^{n}=\sum_{k=0}^{m}\left(\begin{array}{l}
m \\
k
\end{array}\right)\left[(\operatorname{ad} Y)^{k}\left(X^{n}\right)\right] Y^{m-k}
$$

and use the formula ad $Y\left(X^{n}\right)=-n Z X^{n-1}$ to obtain

$$
Y^{m} X^{n}=\sum_{k=0}^{\min (m, n)}(-1)^{k}\left\{\begin{array}{c}
m, n \\
k
\end{array}\right\} Z^{k} X^{n-k} Y^{m-k}
$$

Here

$$
\left\{\begin{array}{c}
m, n \\
k
\end{array}\right\}=\frac{m ! n !}{k !(m-k) !(n-k) !}
$$


We define $\left\{\begin{array}{c}m, n \\ k\end{array}\right\}$ for all integral values of $m, n, k$ by making it zero unless $m \geqq 0$, $n \geqq 0$ and $k \leqq \min (m, n)$. Then (6.1) follows from (6.4) and induction on $n$, with

$$
c_{\alpha, \beta}(k)=(-1)^{|k|}\left\{\begin{array}{c}
\alpha_{1}, \beta_{1} \\
k_{1}
\end{array}\right\} \cdots\left\{\begin{array}{c}
\alpha_{1}+\cdots+\alpha_{n}-\left(k_{1}+\cdots+k_{n-1}\right), \beta_{n} \\
k_{n}
\end{array}\right\}
$$

We rewrite $c_{\alpha, \beta}(k)$ in terms of binomial coefficients:

where

$$
c_{\alpha, \beta}(k)=(-1)^{|k|} \frac{1}{k !} \frac{\alpha !}{(|\alpha|-|k|) !} \frac{\beta !}{(\beta-k) !} d_{\alpha}(k),
$$

$$
d_{\alpha}(k)=\left(\begin{array}{c}
\alpha_{1}+\alpha_{2}-k_{1} \\
\alpha_{2}
\end{array}\right) \cdots\left(\begin{array}{c}
\alpha_{1}+\cdots+\alpha_{n}-\left(k_{1}+\cdots+k_{n-1}\right) \\
\alpha_{n}
\end{array}\right) .
$$

Since $n \rightarrow\left({ }^{a}{ }_{b}^{n}\right)$ is monotone decreasing with $n$, we can majorize $d_{\alpha}(k)$ by $d_{\alpha}(0)$ $=|\alpha| ! / \alpha !$, and thus obtain estimate (6.2). Q.E.D.

Lemma 6.2. Let $\beta \in N^{n}, r>0$. Then

$$
\sum_{0 \leqq k \leqq \beta} \frac{r^{|k|}}{k !} \frac{|\beta-k| !}{(\beta-k) !} \leqq(|\beta| ! / \beta !) \cdot(|\beta|+1) e^{r+1} .
$$

Proof of Lemma 6.2. Introduce the generating function $\left(w \in C^{n}\right)$

$$
\begin{aligned}
& \phi(w)=\sum_{k \in N^{n}} \frac{|k| !}{k !} w^{k}=\left[1-\left(w_{1}+\cdots+w_{n}\right)\right]^{-1} \\
& \psi(w)=\sum_{k \in N^{n}} \frac{r^{|k|}}{k !} w^{k}=\exp r\left(w_{1}+\cdots+w_{n}\right) .
\end{aligned}
$$

Then the sum to be estimated is the coefficient of $w^{\beta}$ in the power series for $\phi(w) \psi(w)$ $=F\left(w_{1}+\cdots+w_{n}\right)$, where $F(z)=(1-z)^{-1} e^{r z}$. Thus the sum equals

$$
\left.(\beta !)^{-1}(d / d z)^{|\beta|} F(z)\right|_{z=0},
$$

which by Cauchy's inequalities is bounded by $(|\beta| ! / \beta !) \cdot \rho^{-|\beta|}(1-\rho)^{-1} e^{r}$, for $0<\rho<1$. Letting $\rho=|\beta| /(|\beta|+1)$ and using the inequality $\left(1+|\beta|^{-1}\right)^{|\beta|} \leqq e$, we obtain the stated inequality. Q.E.D.

Completion of proof of Theorem 6.2. Suppose $f \in \mathscr{A}_{t, \lambda}$. Let

$$
g=\partial \pi^{\lambda}\left(Z^{c} Y^{\alpha_{1}} X^{\beta_{1}} \ldots Y^{\alpha_{n}} X^{\beta_{n}}\right) f .
$$

By Lemma 6.1, if $r<t$,

$$
\|g\| \leqq \frac{|\alpha| ! \beta !|\lambda|^{c}}{r^{|\alpha+\beta|}} \sum_{0 \leqq k \leqq \beta} \frac{\left(|\lambda| r^{2}\right)^{|k|}}{k !} \frac{|\beta-k| !}{(\beta-k) !} \cdot L_{r, \lambda}(f) .
$$

Applying Lemma 6.2, we get

$$
\|g\| \leqq|\alpha| !(|\beta|+1) !|\lambda|^{c} r^{-|\alpha+\beta|} e^{|\lambda| r^{2}+1} L_{r, \lambda}(f) .
$$


Thus if $\rho_{n}$ is defined by equation (1.1) for the representation $\pi^{\lambda}$ and basis $X, Y, Z$, we have by the foregoing

$$
\rho_{n}(f) \leqq \max _{a+b+c=n}\left\{a !(b+1) !|\lambda|^{c} r^{-(a+b)}\right\} \cdot e^{|\lambda| r^{2}+1} L_{r, \lambda}(f) .
$$

The quantity in braces is easily seen to be bounded by $(n+1) !\left[|\lambda|^{n}(n !)^{-1}+r^{-n}\right]$, which implies $E_{s, \lambda}(f) \leqq c(\lambda, s, r) L_{r, \lambda}(f)$ for $s<r$. Q.E.D.

Let $A_{\lambda}$ be the closure of the operator $1-\partial \pi^{\lambda}(\Delta)$. Just as in the case of the regular representation of $\boldsymbol{R}(\S 5)$, the analytic vectors for $A_{\lambda}$ are already entire vectors for $\pi^{\lambda}$ :

Corollary 6.1. Let $f \in L_{2}(R)$ be an analytic vector for $A_{\lambda}$. Then $f$ is an entire vector for $\pi^{\lambda}$. More precisely, there exists a positive constant $\theta(s, t, \lambda)$ such that if $f \in \mathscr{D}\left(e^{t A_{\lambda}}\right)$ for some $t>0$ then for all $s>0$

$$
M_{s, \lambda}(f) \leqq \theta(s, t, \lambda)\left\|e^{t A_{\lambda} f}\right\| .
$$

In particular, the entire vectors for $\pi^{\lambda}$ are dense in $L_{2}(\boldsymbol{R})$.

Proof. Let $S(\sigma), \sigma>0$, be the unitary operator on $L_{2}(R)$ given by $S(\sigma) f(x)$ $=\sigma^{1 / 2} f(\sigma x) . \quad S(\sigma): \mathscr{S} \rightarrow \mathscr{S}$, and $S\left(\sigma^{-1}\right) \partial \pi^{\lambda}(X) S(\sigma)=\sigma(d / d x), \quad S\left(\sigma^{-1}\right) \partial \pi^{\lambda}(Y) S(\sigma)$ $=\sigma^{-1} \lambda(i x)$. Thus for $\sigma=|\lambda|^{1 / 2}$ we have $S\left(\sigma^{-1}\right) \partial \pi^{\lambda}(\Delta) S(\sigma)=|\lambda|\left[(d / d x)^{2}-x^{2}\right]-\lambda^{2} \cdot 1$. From the spectral analysis of the operator $(d / d x)^{2}-x^{2}$ we conclude that $A_{\lambda}$ has simple discrete spectrum $\{1+|\lambda|(2 n+1+|\lambda|)\}$ with normalized eigenfunctions $\phi_{n}^{\lambda}(x)=|\lambda|^{1 / 4} \phi_{n}\left(|\lambda|^{1 / 2} x\right), \phi_{n}$ the Hermite functions.

Let us write $f=\sum a_{n} \phi_{n}^{\lambda}$, and suppose $f \in \mathscr{D}\left(e^{t A_{\lambda}}\right)$ for some $t>0$. (By Proposition 4.1 and the positivity of $A_{\lambda}$ this is equivalent to the condition that $f$ be an analytic vector for $A_{\lambda}$.) Then $\sum\left|a_{n}\right|^{2} e^{4 t n|\lambda|}<\infty$, so by the Schwartz inequality one has a pointwise estimate

$$
|f(z)| \leqq c(\lambda, t)\left\|e^{t A_{\lambda}} f\right\|\left\{\sum_{n=0}^{\infty} e^{-4 t|\lambda| n}\left|\phi_{n}\left(|\lambda|^{1 / 2} z\right)\right|^{2}\right\}^{1 / 2} .
$$

But by $[17$, p. 252], if $z=x+i y$, then

$$
\sum_{n=0}^{\infty} e^{-n t}\left|\phi_{n}(z)\right|^{2}=\frac{e^{2 t}}{(2 \pi \sinh t)^{1 / 2}} \exp \left(-\varepsilon x^{2}+\delta y^{2}\right),
$$

where $\varepsilon=(\cosh t-1) / \sinh t, \delta=(\cosh t+1) / \sinh t$. Hence the eigenfunction expansion for $f$ with respect to $A_{\lambda}$ converges absolutely, uniformly on compact sets in the complex plane, so $f$ is entire. Since $\varepsilon>0,(6.5)$ follows from (6.6) and (6.7). Q.E.D.

REMARKs. The group $G^{c}$ in this case is just the complexification of $G$ as a linear group (where we take $G$ as all matrices

$$
\left[\begin{array}{lll}
1 & a & c \\
0 & 1 & b \\
0 & 0 & 1
\end{array}\right]
$$


with $a, b, c$ real). The representation $\pi_{\omega}^{\lambda}$ of $G^{c}$ on $\mathscr{H}_{\infty}^{\omega}\left(\pi^{\lambda}\right)$ is given by the same formula as the representation $\pi^{\lambda}$ on $\mathscr{H}(\pi)$ (complex translations being defined on $\mathscr{H}_{\infty}^{\omega}\left(\pi^{\lambda}\right)$ by virtue of Theorem 6.2).

7. The " $a x+b$ " group. We next examine the space of analytic vectors for the infinite-dimensional irreducible unitary representations of the " $a x+b$ " group. This is the group $S$ of affine transformations $x \rightarrow a x+b(a>0, b$ real) of $\boldsymbol{R} . S$ can also be realized as the group of matrices of the form

$$
\left(\begin{array}{ll}
a & b \\
0 & a^{-1}
\end{array}\right), \quad a>0, \quad b \text { real, }
$$

and is the semidirect product of the subgroups

$$
A=\left\{\left(\begin{array}{ll}
a & 0 \\
0 & a^{-1}
\end{array}\right) ; \quad a>0\right\}
$$

and

$$
B=\left\{\left(\begin{array}{ll}
1 & b \\
0 & 1
\end{array}\right) ; \quad b \text { real }\right\}
$$

We have $A=\{\exp t H ;-\infty<t<\infty\}$ and $B=\{\exp s E ;-\infty<s<\infty\}$, where

$$
H=\left(\begin{array}{cc}
1 / 2 & 0 \\
0 & -1 / 2
\end{array}\right), \quad E=\left(\begin{array}{ll}
0 & 1 \\
0 & 0
\end{array}\right), \quad \text { and }[H, E]=E
$$

The group $S$ has two infinite-dimensional irreducible unitary representations. We shall denote them by $\sigma_{ \pm}$and realize them on $L^{2}(R)$ as follows:

$$
\sigma_{ \pm}(g) f(x)=\exp \left( \pm i s e^{x}\right) f(x+t),
$$

where $f \in L_{2}, g=\exp s E \exp t H$ (cf. [8] and [16, §14]). Then $d \sigma_{ \pm}(H)=d / d x$ and $d \sigma_{ \pm}(E)=$ multiplication by $\pm i e^{x}$, with the usual maximal domains as skew-adjoint operators. By Theorem 1.1 the space $\mathscr{H}^{\infty}\left(\sigma_{ \pm}\right)$consists of the $C^{\infty}$ functions $f$ with $f^{(n)}(x)$ and $e^{n x} f(x)$ in $L_{2}$ for $n=0,1, \ldots$ (Such a function then having the property that $e^{n x} f^{(m)}(x)$ is in $L_{2}$ for all positive $m$ and $n$.) Notice that the second condition is a restriction only at $x=+\infty$.

Consider the operator $\partial \sigma_{ \pm}\left(H^{2}+E^{2}\right)=d^{2} / d x^{2}-e^{2 x}$, acting on the space $\mathscr{H}^{\infty}\left(\sigma_{ \pm}\right)$ just described. Let $-T$ be the closure of this operator. $T$ is then a selfadjoint, nonnegative operator (selfadjointness follows, e.g. by [21, Theorem 2.2]), and in fact the eigenfunction expansion associated with $T$ has been explicitly calculated in [29, pp. 95-96]. It turns out that the unitary operator diagonalizing $T^{1 / 2}$ is essentially the "Lebedev transform" [15], [17, p. 398]. Thus our Theorem A yields a theorem of Paley-Wiener type relating analyticity properties of a function to the exponential decrease of its Lebedev transform.

To elaborate, we first sketch the derivation of the eigenfunction expansion formula for $T$, referring to [6] for the underlying theory. 
One calculates that for $\operatorname{Im} \lambda \neq 0,(T+\lambda)^{-1}$ is an integral operator with kernel (relative to Lebesgue measure)

$$
\begin{aligned}
G_{\lambda}(x, \xi) & =K_{\sqrt{ } \lambda}\left(e^{x}\right) I_{\sqrt{ } \lambda}\left(e^{\xi}\right), & \xi<x, \\
& =I_{\sqrt{ } \lambda}\left(e^{x}\right) K_{\sqrt{ } \lambda}\left(e^{\xi}\right), & \xi>x .
\end{aligned}
$$

Here $K_{v}$ and $I_{v}$ are the modified Bessel functions [17, p. 66] and $\sqrt{ } \lambda=|\lambda|^{1 / 2} e^{i \theta / 2}$, $\arg \lambda=\theta \quad(-\pi<\theta<\pi)$. Using the Stieltjes inversion formula [6, Theorem XII, 2.10] and formulas relating $K_{ \pm v}(x)$ and $I_{ \pm v}(x)$ [17, p. 66], one obtains the following expression for the spectral projection $E([a, b])$ of $T$ :

$$
(E([a, b]) f \mid g)=\frac{1}{\pi^{2}} \int_{a}^{b} U f(\lambda) \overline{U g(\lambda)} \sinh \pi \sqrt{ } \lambda d \lambda .
$$

Here $0 \leqq a \leqq b$, and

$$
U f(\lambda)=\int_{-\infty}^{\infty} K_{i \sqrt{ } \lambda}\left(e^{x}\right) f(x) d x
$$

defines a unitary map from $L_{2}(R)$ to $L_{2}\left(R^{+} ; \pi^{-2} \sinh \pi \sqrt{ } \lambda d \lambda\right)$. By (7.2) we have $U T U^{-1}=$ multiplication by $\lambda$, and the eigenfunction expansion for $T$ is

$$
f(x)=\pi^{-2} \int_{0}^{\infty} K_{i \sqrt{ } \lambda}\left(e^{x}\right) U f(\lambda) \sinh \pi \sqrt{ } \lambda d \lambda
$$

$[29$, p. 96$]$.

To obtain the eigenfunction expansion for $T^{1 / 2}$ we simply make the change of variable $\nu=\sqrt{ } \lambda$ in (7.2), (7.3), and (7.4). Thus defining

$$
f(\nu)=\lim \int_{-\infty}^{\infty} K_{i v}\left(e^{x}\right) f(x) d x, \quad \nu>0,
$$

we have $\left(T^{1 / 2} f\right) \sim(\nu)=\nu \tilde{f}(\nu)$ and

$$
f(x)=\lim \int_{0}^{\infty} K_{i v}\left(e^{x}\right) \tilde{f}(v) d \mu(\nu)
$$

$d \mu=2 \pi^{-2} \nu \sinh \pi \nu d \nu$. We shall call $f$ the Lebedev transform of $f$. (This differs from $\left[17\right.$, p. 398] by the change of variable $y=e^{x}$.)

REMARKS. For a general $f \in L_{2}(\boldsymbol{R})$, formulas (7.5) and (7.6) have to be interpreted in the usual $L_{2}$-limit sense. Using the integral representation

$$
K_{i v}(x)=\int_{0}^{\infty} e^{-x \cosh t} \cos (\nu t) d t, \quad x>0
$$

$\left[17\right.$, p. 85] and the inequality $\cosh t \geqq 1+\frac{1}{2} t^{2}$, one obtains the estimate

$$
\left|K_{i v}\left(e^{x}\right)\right| \leqq C \exp \left[-\frac{1}{2} x-e^{x}\right]
$$

Hence if $f$ is $O\left(\exp -\left(\frac{1}{2}+\varepsilon\right)|x|\right)$ at $-\infty$, for example, then (7.5) is absolutely convergent. The inversion formula (7.6) admits a slightly neater treatment: Since the 
estimate (7.8) is independent of $\nu$, integral (7.6) is absolutely convergent for $f=e^{-\varepsilon T} g, g \in L_{2}(\boldsymbol{R}), \varepsilon>0$. Of course $e^{-\varepsilon T} g \rightarrow g$ in $L_{2}$ as $\varepsilon \rightarrow 0$.

We can now give the Paley-Wiener type of characterization to the space of analytic vectors for the representations $\sigma_{ \pm}$.

THEOREM 7.1. Let $f \in L_{2}(R)$. The following are equivalent:

(i) $f$ is an analytic vector for the representation $\sigma_{+}$or $\sigma_{-}$of the group $S$.

(ii) The Lebedev transform $\tilde{f}$ of $f$ satisfies

$$
\int_{0}^{\infty}|\tilde{f}(\nu)|^{2} e^{\sigma \nu} \nu \sinh \pi \nu d \nu<\infty
$$

for some $\sigma>0$.

(iii) $f \in \mathscr{H}_{t}^{\omega}(U)$ and satisfies

$$
\sup _{|y|<t}|f(x+i y)| \leqq C e^{-\sigma e^{x}},
$$

for some $C, \sigma, t>0$. (Cf. $\S 5$ for the space $\mathscr{H}_{t}^{\omega}(U)$.)

Proof. (i) $\Leftrightarrow$ (ii) follows from the above discussion and Theorem A of the Introduction.

(i) $\Leftrightarrow$ (iii) follows exactly as in the proof of Theorem 6.1 , since $S$ is the semidirect product of the subgroups $A$ and $B$.

The next theorem shows that, in contrast to the respresentations $\pi^{\lambda}$ of the Heisenberg group, the representations $\sigma_{ \pm}$of the group $S$ do not admit any nonzero entire vectors, in any sense.

THEOREM 7.2. Let $\sigma$ be a unitary representation of $S$ of the form $\sigma=C_{+} \sigma_{+} \oplus C_{-} \sigma_{-}$, where $C_{ \pm}$are cardinal numbers, and let $v \in \mathscr{H}(\sigma)$. Suppose that

(i) $g \mapsto(\sigma(g) v, v)$ is analytic on $S$.

(ii) $\phi(t)=(\sigma(\exp t H) v, v)$ extends holomorphically to the complex disc $|t|<t_{0}$ for some $t_{0}>2 \pi$. Then $v=0$.

REMARK. An arbitrary unitary representation of $S$ is of the form $C_{+} \sigma_{+} \oplus C_{-} \sigma_{-}$ $\oplus \tau$, where $\tau$ is a direct integral of one-dimensional representations [8]. Thus by spectral theory $\tau$ always has a dense set of entire vectors. Theorem 7.2 asserts that this is the only source of entire vectors for representations of $S$.

Proof of Theorem 7.2. By Proposition 4.1 applied to the one-parameter unitary groups $t \rightarrow \sigma(\exp t H)$ and $t \rightarrow \sigma(\exp t E)$, conditions (i) and (ii) on $v$ imply that $v$ is an analytic vector for the subgroups $A$ and $B$ of $S$, hence an analytic vector for $S$. Furthermore we have estimates $\left\|\partial \sigma\left(H^{m}\right) v\right\| \leqq M m ! r^{-m}$ and $\left\|\partial \sigma\left(E^{n}\right) v\right\| \leqq M n ! s^{-n}$ for some $r>\pi$ and $s>0$. In order to get an explicit estimate for $\left\|\partial \sigma\left(H^{m} E^{n}\right) v\right\|$, we observe that the commutation relation $[H, E]=E$ implies ad $H\left(E^{n}\right)=n E^{n}$. Hence by identity $(6.3)$,

$$
H^{m} E^{n}=\sum_{k=0}^{m}\left(\begin{array}{c}
m \\
k
\end{array}\right) n^{k} E^{n} H^{m-k}
$$


Thus using the skew-symmetry of $\partial \sigma(H)$ and $\partial \sigma(E)$ together with equation (7.9), we obtain the estimate

$$
\begin{aligned}
\left\|\partial \sigma\left(H^{m} E^{n}\right) v\right\|^{2} & =(-1)^{m+n}\left(\partial \sigma\left(E^{n} H^{2 m} E^{n}\right) v, v\right) \\
& =(-1)^{m+n} \sum_{k=0}^{2 m}\left(\begin{array}{c}
2 m \\
k
\end{array}\right) n^{k}\left(\partial \sigma\left(H^{2 m-k}\right) v, \partial \sigma\left(E^{2 n}\right) v\right) \\
& \leqq M^{2}(2 m) !(2 n) ! r^{-2 m} S^{-2 n} \cdot \sum_{k=0}^{2 m}(n r)^{k} / k !
\end{aligned}
$$

which gives

$$
\left\|\partial \sigma\left(H^{m} E^{n}\right) v\right\| \leqq M m ! n !(r / 2)^{-m}\left(s / 2 e^{r}\right)^{-n} .
$$

If we now decompose $v=\sum \oplus v_{k}^{ \pm}$according to the direct-sum decomposition $\mathscr{H}(\sigma)=C_{+} \mathscr{H}\left(\sigma_{+}\right) \oplus C_{-} \mathscr{H}\left(\sigma_{-}\right)$, then each $v_{k}^{ \pm} \in \mathscr{H}^{\omega}\left(\sigma_{ \pm}\right)$and satisfies estimate (7.10). Thus it suffices to treat the cases $\sigma=\sigma_{ \pm}$. But in this case (cf. the proof of Theorem 6.1), estimate (7.10) implies that $v$ is the restriction to the real axis of a function $f(z)$, holomorphic in the strip $|\operatorname{Im} z|<r / 2$ and satisfying

$$
\sup _{|y| \leqq \rho}\left|\exp \left(\varepsilon e^{x}\right) f(x+i y)\right| \leqq M_{\rho}
$$

for some $\varepsilon>0$ and any $\rho<r / 2$. Since $r / 2>\pi / 2$, this however forces $f \equiv 0$, by a theorem of Phragmén-Lindelöf type.

Indeed, by (7.11) and the maximum modulus theorem

$$
\sup _{|\operatorname{Im} z| \leqq \rho}\left|\exp \left(\delta e^{\gamma z}\right) f(z)\right| \leqq M_{\rho}
$$

for any $\delta>0$, where $\gamma=\pi / 2 \rho$ and $\rho>\pi / 2$. Let $\delta \rightarrow+\infty$. (Cf. [23, problem 326].) Q.E.D.

Define the spaces $\mathscr{H}_{t}^{\omega}(\sigma)$ relative to the basis $\{H, E\}$ for the Lie algebra of $S$. Then Theorem 7.2 and Proposition 4.1 imply

COROLLARY 7.1. $\mathscr{H}_{t}^{\omega}(\sigma)=0$ for $t>\pi$.

RemarKs. 1. Observe that the formula (7.1), when $f$ is an entire function, is defined for arbitrary complex values of $s$ and $t$, just as in the case of the formula for the representations $\pi^{\lambda}$ of the Heisenberg group. However, the representations of $S^{c}$ thus obtained leave invariant no nonzero subspace of the space of analytic vectors for $S$. For example, the entire function $f(z)=\exp \left[z-e^{z}\right]$ is in $\mathscr{H}^{\omega}\left(\sigma_{ \pm}\right)$by Theorem 7.1 , but its complex translates do not remain in $L_{2}$.

2. The argument leading to estimate (7.10) gives an alternate proof of Theorem 3.1 for unitary representations of the group $S$. (In deriving (7.10) we did not use the fact that $v \in \mathscr{H}^{\omega}(\sigma)$; it sufficed to know that $v \in \mathscr{H}^{\infty}(\sigma)$, which was a consequence of Theorem 1.1.) A similar argument may be employed in connection with the Heisenberg group, and leads to a considerable strengthening of Corollary 3.1 for unitary representations of this group. 
8. Noncompact simple groups. Let $G$ be a connected noncompact Lie group whose Lie algebra $\mathfrak{g}$ is simple. Take a Cartan decomposition $\mathfrak{g}=\mathfrak{t}+\mathfrak{p}$ (since $G$ is noncompact $\mathfrak{p} \neq 0$ ), and let $\mathfrak{a}$ be a maximal abelian subspace of $\mathfrak{p}$, with $R \subseteq \mathfrak{a}^{\prime}$ the nonzero roots of $g$ relative to $a$, [10, Chapter VI]. The Killing form $B$ is positivedefinite on $\mathfrak{a}$, and hence defines norms on $\mathfrak{a}^{\prime}$ and on $\mathfrak{a}^{c}$ which will be denoted by $\|\cdot\|$. Define a positive number $r_{0}$ by

$$
r_{0}=\inf _{\lambda \in R}\|\lambda\| .
$$

Suppose now that $\pi$ is a unitary representation of $G$ which does not contain the identity representation. The following theorem shows that a nonzero matrix element of $\pi$ which is analytic on $G$ must have a singularity on $\mathfrak{a}^{c}$, within a predictable distance from $a$.

TheOREM 8.1. Let $v \in \mathscr{H}(\pi)$ and set $\phi(g)=(\pi(g) v, v)$. Suppose that

(i) $\phi$ is an analytic function on $G$.

(ii) The function $X \mapsto \phi(\exp X)$ on a extends holomorphically to the ball $\|X\|<$ $2 \pi r_{0}+\varepsilon$ in $\mathfrak{a}^{c}$ for some $\varepsilon>0$.

Then $v=0$.

We first establish a preliminary lemma suggested by Lemma 4 of [26], which is of some independent interest (and is probably not new).

Lemma 8.1. Let $X \in \mathfrak{p}, X \neq 0$. Then the one-parameter unitary group $P(t)=$ $\pi(\exp t X)$ has a continuous spectrum. In particular, $P(t)$ leaves fixed no nonzero vector in $\mathscr{H}(\pi)$.

Proof. Let $\mathscr{H}_{\mu}=\left\{v \in \mathscr{H} \mid P(t) v=e^{i t \mu} v\right\}, \mu$ real. Since $X \in \mathfrak{p}$, ad $X$ is real-diagonalizable; let $Y \in \mathfrak{g}$ satisfy $[X, Y]=\lambda Y$. If $\lambda=0$, then $\pi(\exp t Y)$ leaves $\mathscr{H}_{\mu}$ invariant. If $\lambda \neq 0$, then $H=\lambda^{-1} X$ satisfies $[H, Y]=Y$. By the representation theory of the group $S$ of $\S 7$ (cf. [8]), we must have $\pi(\exp t Y) v=v$ for all $v \in \mathscr{H}_{\mu}$. Thus $\mathscr{H}_{\mu}$ is invariant under $G$, since $G$ is connected. Furthermore, since ad $X \neq 0$, we also conclude that $G^{\mu} \equiv \operatorname{Ker}\left(\left.\pi\right|_{\mathscr{H}_{\mu}}\right)$ contains a one-parameter subgroup. Since the only normal subgroups of $G$ are discrete, this implies that $G^{\mu}=G$. Hence $\mathscr{H}_{\mu}=0$, since $\pi$ does not contain the identity representation. Q.E.D.

Proof of Theorem 8.1. Let

$$
\mathfrak{g}=\mathfrak{g}_{0}+\sum_{\alpha \in R} \mathfrak{g}_{\alpha}
$$

be the weight-space decomposition of $\mathfrak{g}$ with respect to $\mathfrak{a}$ [10, Chapter VI]. For every $\alpha \in R$, if $H_{\alpha} \in \mathfrak{a}$ is determined by $B\left(H, H_{\alpha}\right)=\alpha(H) /\|\alpha\|^{2}$, all $H \in \mathfrak{a}$, then there exist $E_{\alpha}$ and $F_{\alpha}$ in $g$ satisfying the commutation relations $\left[H_{\alpha}, E_{\alpha}\right]=E_{\alpha},\left[H_{\alpha}, F_{\alpha}\right]$ $=-F_{\alpha},\left[E_{\alpha}, F_{\alpha}\right]=H_{\alpha}$ (cf. [10, Chapter VII, Lemma 2.3]). Let $G_{\alpha}$ be the connected subgroup of $G$ with Lie algebra spanned by $\left\{H_{\alpha}, E_{\alpha}, F_{\alpha}\right\}\left(G_{\alpha}\right.$ is locally isomorphic to $\operatorname{SL}(2, \boldsymbol{R}))$. Since $\left\{\pi\left(\exp t H_{\alpha}\right)\right\}$ fixes no nonzero vector in $\mathscr{H}(\pi)$, by Lemma 8.1, 
we know that $\pi_{\alpha}=\left.\pi\right|_{G_{\alpha}}$ does not contain the identity representation of $G_{\alpha}$. If $S_{\alpha}$ is the subgroup of $G_{\alpha}$ with Lie algebra spanned by $\left\{H_{\alpha}, E_{\alpha}\right\}$, then $S_{\alpha} \cong S$. Set $\sigma_{\alpha}=\left.\pi\right|_{S_{\alpha}}$. Then by [26, Lemma 2], $\sigma_{\alpha}$ is of the form $C_{+} \sigma_{+} \oplus C_{-} \sigma_{-}$, where $C_{ \pm}$are cardinal numbers and $\sigma_{ \pm}$are the representations of $S$ discussed in $\$ 7$. Theorem 8.1 now follows immediately from Theorem 7.2 applied to the group $S_{\alpha}$ for which $\|\alpha\|=r_{0}$. (Note that $\left\|H_{\alpha}\right\|=1 /\|\alpha\|$.)

As an immediate consequence of Theorem 8.1 and Corollary 2.1 we have

COROllary 8.1. $\mathscr{H}_{t}^{\omega}(\pi)=0$ for $t$ sufficiently large (independent of. $\pi$ ).

We also obtain the following result, due to I. M. Singer:

COROLlaRY 8.2. $g \mapsto \pi(g)$ cannot be continuous in the uniform operator topology.

Proof. If it were, then $\partial \pi(X)$ would be a bounded operator for all $X \in \mathfrak{g}$. Hence every vector would be an entire vector, contradiction.

REMARK. In Singer's proof of this result [27], as well as in earlier related work of Segal and von Neumann [25], the embedding of the group $S$ in $G$ also plays a key role.

Suppose now that $G$ has a finite center, and let $K$ be the subgroup corresponding to $\mathfrak{f}$. $K$ is compact, and one says that a vector $v \in \mathscr{H}(\pi)$ is $K$-finite in case span $\{\pi(K) v\}$ is finite-dimensional. Since every $K$-finite vector defines an analytic matrix element [7, Lemma 17], we have

Corollary 8.3. If $v$ is $K$-finite, then the function $X \rightarrow(\pi(\exp X) v, v), X \in \mathfrak{a}$, cannot be analytically continued to the ball $\|X\|<2 \pi r_{0}+\varepsilon$ in $\mathfrak{a}^{c}$ for any $\varepsilon>0$.

Example. Let $G=\operatorname{SL}(2, C)$. The matrices

$$
H=\left(\begin{array}{cc}
1 / 2 & 0 \\
0 & -1 / 2
\end{array}\right), \quad E=\left(\begin{array}{ll}
0 & 1 \\
0 & 0
\end{array}\right) \text { and } \quad F=\left(\begin{array}{ll}
0 & 0 \\
1 & 0
\end{array}\right)
$$

in $\mathfrak{g}$ satisfy $[H, E]=E,[H, F]=-F,[E, F]=H$. Let $\pi$ be the representation $\sigma_{0, \rho}$ of the principal series for $G$ [19], and $v$ a $K$-fixed unit vector in $\mathscr{H}(\pi)$. Corollary 8.3 predicts that the function $\phi(t)=(\pi(\exp t H) v, v)$ cannot be analytically continued beyond the disc $|t|<2 \pi$. On the other hand, from [19, §11.5, formula 11], one has

$$
\phi(t)=\frac{2}{\rho} \frac{\sin (\rho t / 2)}{\sinh (t / 2)} .
$$

Thus $\phi$ has poles at $t= \pm 2 \pi i$, so Corollary 8.3 is best possible.

\section{REFERENCES}

1. L. Bers, F. John and M. Schechter, "Partial differential equations" in Lectures in applied mathematics, Vol. III, Interscience, New York, 1964.

2. R. J. Blattner, On induced representations, Amer. J. Math. 83 (1961), 79-98.

3. F. Browder, Real analytic functions on product spaces and separate analyticity, Canad. J. Math. 13 (1961), 650-656. 
4. F. Bruhat, Sur les représentations induites des groupes de Lie, Bull. Soc. Math. France 84 (1956), 97-205.

5. P. Cartier, Représentations des groupes de Lie, Séminaire Bourbaki \#96 (1954); reprint, Benjamin, New York, 1967.

6. N. Dunford and J. Schwartz, Linear operators, Vol. II, Interscience, New York, 1963.

7. R. Godement, A theory of spherical functions. I, Trans. Amer. Math. Soc. 73 (1952), 496-556.

8. I. Gelfand and M. Naimark, Unitary representations of the group of linear transformations of the straight line, Dokl. Akad. Nauk SSSR 55 (1947), 567-570.

9. R. Goodman, Analytic domination by fractional powers of a positive operator, J. Functional Analysis 3 (1969), 246-264.

10. S. Helgason, Differential geometry and symmetric spaces, Academic Press, New York, 1962.

11. E. Hille, Contributions to the theory of Hermitian series. II. The representation problem, Trans. Amer. Math. Soc. 47 (1940), 80-94.

12. G. Hochschild, The structure of Lie groups, Holden-Day, San Francisco, Calif., 1965.

13. Y. Katznelson, An introduction to harmonic analysis, Wiley, New York, 1968.

14. R. Kunze and I. E. Segal, Integrals and operators, McGraw-Hill, New York, 1968.

15. N. N. Lebedev, Sur une formule d'inversion, Dokl. Akad. Nauk SSSR 52 (1946), 655-658.

16. G. W. Mackey, Induced representations of locally compact groups. I, Ann. of Math. 55 (1952), 101-139.

17. W. Magnus, F. Oberhettinger and R. P. Soni, Formulas and theorems for the special functions of mathematical physics, Springer, New York, 1966.

18. R. T. Moore, Measurable, continuous, and smooth vectors for semigroups and group representations, Mem. Amer. Math. Soc. No. 78, 1968.

19. M. A. Naimark, Linear representations of the Lorentz groups, Macmillan, New York, 1964.

20. E. Nelson, Analytic vectors, Ann. of Math. 70 (1959), 572-615.

21. E. Nelson and W. F. Stinespring, Representation of elliptic operators in an enveloping algebra, Amer. J. Math. 81 (1959), 547-560.

22. R. E. A. C. Paley and N. Wiener, Fourier transforms in the complex domain, Amer. Math. Soc. Colloq. Publ., Vol. 19, Amer. Math. Soc., Providence, R. I., 1934; reprinted 1967.

23. G. Pólya and G. Szegö, Aufgaben und Lehrsätze aus der Analysis. I, Springer, Berlin, 1954.

24. L. Pukanszky, Leçons sur les représentations des groupes, Dunod, Paris, 1967.

25. I. E. Segal and J. von Neumann, A theorem on unitary representations of semi-simple Lie groups, Ann. of Math. 52 (1950), 509-517.

26. T. Sherman, A weight theory for unitary representations, Canad. J. Math. 18 (1966), 159-168.

27. I. M. Singer, Uniformly continuous representations of Lie groups, Ann. of Math. 56 (1952), 242-247.

28. E. M. Stein, Analytic continuation of group representations, Lectures, Yale University, 1967.

29. E. C. Titchmarsh, Eigenfunction expansions, Part I, 2nd ed., Oxford Univ. Press, Oxford, 1962.

Massachusetts Institute of Technology, CAMbridge, Massachusetts

INSTITUTE for AdVANCEd StUdy, Princeton, New Jersey 